\title{
Cardiac Troponin-I Status of Type-2 Diabetic Patients on Anti-Diabetic Drugs Treatment at Jimma Medical Center, Jimma, Southwest Ethiopia
}

\author{
Alemayehu Babusha Wega $\mathbb{D}^{\prime}$ \\ Endriyas Kelta Wabalo ${ }^{2}$ \\ Chala Kenenisa Edae (iD ${ }^{2}$ \\ Gesese Bogale Awgichew ${ }^{2}$ \\ 'Department of Biomedical Sciences, \\ College of Medicine and Health Sciences, \\ Mettu University, Mettu, Ethiopia; \\ ${ }^{2}$ Department of Biomedical Sciences, \\ Faculty of Medical Sciences, Institute of \\ Health, Jimma University, Jimma, Ethiopia
}

Introduction: Metabolic abnormalities of type-2 diabetes mellitus cause vascular dysfunction which is manifested with common cardiovascular complications such as atherosclerosis, myocardial infarction (MI), stroke, and coronary heart diseases. In spite of all these complications, there is no literature revealing the cardiac status of type- 2 diabetic patients, in general and on different anti-diabetic drugs in particular, using cardiac troponin-I as a biomarker in study area and Ethiopia too.

Objective of the Study: This study was, therefore, aimed to assess cardiac troponin-I in type-2 diabetic patients on anti-diabetic drugs treatment at Jimma Medical Center, Jimma, Southwest Ethiopia.

Methods: To achieve the objectives of the study, a facility based cross-sectional study was conducted among type-2 diabetic patients on follow-up at Jimma Medical Center from August 1 to September 30, 2020. Systematic sampling technique was used to select 82 study participants. A $5 \mathrm{~mL}$ of venous blood was drawn from each participant after overnight fasting using an aseptic technique and centrifuged at $3000 \mathrm{rpm}$ for 10 minutes. Multiple logistic regression analysis and one way ANOVA were used for statistical data analysis. P-value $<0.05$ was considered as statistically significant.

Results: The prevalence of elevated cardiac troponin-I was $25.6 \%$ in the study population. The mean value of serum cardiac troponin-I was lower in patients who were on metformin monotherapy as compared to patients on insulin monotherapy $(11.654 \pm 1.6795$ versus $20.573 \pm$ $1.8402, \mathrm{P}<0.01)$. It was also lower when compared with those patients on metformin + glibenclamide combination therapy $(11.654 \pm 1.6795$ versus $21.094 \pm 2.4062, \mathrm{P}<0.01)$.

Conclusion and Recommendations: About $1 / 4^{\text {th }}$ of the study participants had elevated serum cardiac troponin-I level. Metformin monotherapy significantly reduced serum cTnI levels as compared to insulin monotherapy and metformin + glibenclamide combination therapy. Regular screening for cardiac injury using cardiac troponin-I is recommended for type- 2 diabetic patients. Keywords: cardiac troponin-I, type-2 diabetes mellitus, anti-diabetic drugs, Jimma Medical Center, Ethiopia

\section{Introduction}

Diabetes mellitus is a chronic metabolic disease characterized by elevated blood glucose due to insulin resistance or insulin deficiency. This elevated blood sugar is responsible for triggering symptoms such as polyuria, polydipsia, and polyphagia. ${ }^{1}$

Diabetes mellitus can be classified into four types: type-1 diabetes, type-2 diabetes, gestational diabetes mellitus, and other specific types of diabetes. Type1 and type- 2 diabetes mellitus are the most common forms of diabetes mellitus. ${ }^{2}$ 
According to the International Diabetes Federation (IDF) report, type-2 diabetes became the leading type among all cases of diabetes mellitus, contributing to $90 \%$ to $95 \%$ of cases in developed countries and may exceed this percentage in developing countries due to urbanization and westernization. ${ }^{3}$

Type-2 diabetes mellitus is associated with increased risk for cardiovascular disease, probably due to its nature of pathogenesis and coexisting cardiovascular risk factors like hypertension, obesity, and dyslipidemia. ${ }^{2}$ Vascular dysfunction caused by metabolic abnormalities in patients with type- 2 diabetes is associated with accelerated atherosclerosis and increased risk of myocardial infarction (MI), stroke, and peripheral arterial disease. It is also a strong risk factor for coronary artery disease, and patients with diabetes have two to four-fold greater risk of developing coronary artery disease., ${ }^{2,4}$

Cardiac troponin is one of the cardiac biomarkers used to diagnose myocardial injury both in symptomatic and asymptomatic individuals. ${ }^{5}$ Troponin is a protein complex made up of three subunits namely troponin- $\mathrm{T}(\mathrm{TnT})$, troponin-I ( $\mathrm{TnI}$ ), and troponin-C ( $\mathrm{TnC})$, each having different structure and function and located on the thin filament of striated muscles. These three subunits of troponin complex along with tropomyosin is located on the actin filament and is essential for the calcium-mediated regulation of skeletal and cardiac muscle contraction. ${ }^{6,7}$

Among the three troponins, $\mathrm{TnT}$ and $\mathrm{TnI}$ are being used as the biochemical markers for the diagnosis of myocardial injury. The amino acid sequence of cardiac troponin- $T$ and troponin-I is different from that present in skeletal muscles. This is a base for cardiac troponin specificity in the diagnosis of myocardial injury. ${ }^{7}$ Normally, about $94-97 \%$ of $\mathrm{TnT}$ and $\mathrm{TnI}$ are bound to myofibril and only $6 \%$ of cTnT and $3 \%$ of the cTnI are free in the cytoplasm, but during myocardial damage, the cytosolic troponin reaches the bloodstream quickly resulting in a rapid peak of serum troponin observed during the first few hours. Next, the release of structurally bound troponin results in a second peak lasting for several days. These detectable serum levels of cTns are an indicator of cardiac muscle damage. $^{8,9}$

Compared to other available cardiac markers, cardiac troponin-I has better sensitivity and tissue specificity and it detects myocardial injury. Following myocardial cell injury caused by myocardial ischemia, the myocardial cell membrane integrity is compromised and free cardiac
troponin-I from myocardial cell cytoplasm is rapidly released into the blood circulation. ${ }^{10}$

In order to improve the glycemic control and reduce the risk of diabetic cardiovascular complications both pharmacological and non-pharmacological treatment modalities (eg lifestyle modifications) are recommended for diabetic patients. ${ }^{11}$ Metformin, insulin secretagogues, and acarbose are first-line oral glucose control therapies in type- 2 diabetic patients. Whereas dipeptidyl peptidase- 4 inhibitor, a thiazolidinedione, and glucagon-like peptide-1 receptor agonists are second-line drugs introduced when blood glucose levels cannot be controlled by first-line drugs or when patients are unable to tolerate those drugs. The final stage is insulin, which is used alone or in combination with other oral hypoglycemic drugs in case blood glucose level control cannot be achieved. ${ }^{11,12}$

In sub-Saharan Africa, the average number of adults with type-2 diabetic cases was 15.5 million in 2017 , with a regional prevalence of approximately $6 \%$, and this number is estimated to increase by $162.5 \%$ by $2045 .{ }^{14}$ Like other sub-Saharan African countries, Ethiopia is suffering from a high burden of diabetes, with increased prevalence, complications, and mortality and life-threatening disabilities. According to a report of World Health Organization (WHO) in 2000, the number of diabetes cases in Ethiopia was 800,000 . Meanwhile, this number is estimated to rise to 1.8 million by the year $2030 .{ }^{14,15}$

The World Health Organization's report revealed that a trend in the prevalence of diabetes in Ethiopia has been increasing over the past years. According to the 2017 International Diabetes Federation (IDF), the prevalence of type-2 diabetes mellitus in Ethiopian adults was 5.2\%. ${ }^{13}$

Although the prevalence of type- 2 diabetes mellitus is increased and accompanied by cardiovascular complications among Ethiopian population, ${ }^{16-19}$ there are no data showing cardiac status of these patients using cardiac troponin-I as a biomarker to the researcher's knowledge. In addition, it has not been determined whether antidiabetic drugs have different cardiovascular effects in type-2 diabetic patients in the Ethiopian population. Therefore, the purpose of this study was to assess the cardiac status using cardiac troponin-I and compare the effects of different anti-diabetic drugs on cardiac troponinI in the study area.

\section{Methods and Materials}

This study was conducted at Jimma Medical Center (JMC) from August 1 up to September 30, 2020. JMC is located 
in Jimma town at a distance of $352 \mathrm{~km}$ to the Southwest of Addis Ababa. It is the only teaching and referral hospital in the South-Western part of the country with a bed capacity of 600 .

A facility based cross-sectional study was conducted during the study period.

All type-2 diabetic patients on anti-diabetic drug treatment and on follow-up at the chronic illness clinic of JMC were considered as a source population and all sampled type-2 diabetic patients on anti-diabetic drug treatment and on follow-up at the chronic illness clinic of JMC who fulfilled the inclusion criteria were taken as the study population.

Type-2 diabetic patients on anti-diabetic drug treatment and on follow-up at the chronic illness clinic of JMC who were 18 and above were included in the study. On the other hand, patients who have chronic kidney disease, were critically sick and unable to communicate, were on lipid lowering drugs and chemotherapy and pregnant women were excluded from the study.

The sample size of 86 was calculated by using single population proportion formula: $\mathrm{n}=\left(\mathrm{Z}_{1-\alpha / 2}\right)^{2} \mathrm{P}(1-\mathrm{P}) / \mathrm{d}^{2}$, using $\mathrm{p}=26 \%$ prevalence of elevated troponin in USA (20) and population correction formula $\mathrm{nf}=\mathrm{n} / 1+\mathrm{n} / \mathrm{N}$ and adding $10 \%$ of non-response rate.

A systematic sampling technique was used. The $\mathrm{k}$-value was calculated by dividing the total population by the sample size, $K=2700 / 82=32.926=33$. Data were collected from every 33 individuals starting from the first arrived patients at the beginning of data collection.

Serum cardiac troponin-I level was taken as dependent variable and anti-diabetic drugs were considered as independent variables.

Five milliliters of venous blood sample was collected from study participants after overnight fasting using an aseptic technique and centrifuged after 30 minutes at $3000 \mathrm{rpm}$, for 10 minutes. Separated serum was kept at $-20^{\circ} \mathrm{C}$ in the refrigerator till used. The serum cardiac troponin-I were measured using ARCHITECT c4000 highsensitive troponin-I assay (Abbott Laboratories, Abbott Park, IL, USA) random access full automated auto analyzer.

Data quality was assured during blood sample collection by strictly following the standard aseptic operational procedure. The kit was made free from contamination and check for consistency. Laboratory analysis was done following the appropriate procedures based on the manufacturer's instruction. All the laboratory procedures were handled with the assistance of professional laboratory technologists and results were checked for completeness to maintain the overall quality of data. During data entry and analysis using computer software, due attention was given to keep the data quality.

All data were checked, cleared, and fed into Epi-data (version 3.1) and then exported to SPSS (version 25.0) software for statistical analysis. After the complete entry of all the data, a soft copy was checked with its hard copy to see the consistency. The data were also checked for the fulfillment of assumption. It was processed by using descriptive analysis, including frequency distribution. Comparison of mean difference of serum cardiac troponin-I among different types of anti-diabetic drugs was done using one way ANOVA after checking its assumption.

\section{Ethical Considerations}

Before starting the research, ethical review committee of Jimma University approved this research project and a letter of ethical clearance was obtained with protocol Number IHRPGD 715/20. Then formal letter was obtained from Jimma University institute of health and taken to the chief clinical director of JMC and the permission letter was offered.

Next, the permission letter was given to the chronic follow-up clinic to conduct the study. To prevent COVID19, data collectors used personal protective equipment like surgical masks, disposable gloves, and hand sanitizer. Additionally, the distance between data collectors and study participants was kept at least $2 \mathrm{~m}$ apart during face to face interview. Study participants were also encouraged to use a face mask and hand sanitizer as per the national protocol of COVID-19 prevention strategies. During data collection, only one participant was allowed in a room at a time.

All study participants were informed about the research; the right to withdraw themselves at any time and confidentiality of information to be maintained during data collection, analysis, interpretation, and publication of results. The written consent was granted from each study participants based on their interest.

The confidentiality of the data was insured by using code rather than patients name and all the data results that showed deviation from normal reference was reported to physicians working in chronic disease follow up clinic. 


\section{Operational Definitions}

Elevated cardiac troponin: Elevated cardiac troponin was considered as a serum cardiac troponin-I level $>34.2 \mathrm{pg} /$ $\mathrm{mL}$ for male and $>15.6 \mathrm{pg} / \mathrm{mL}$ for female at 99th percentile upper reference limit.

\section{Result}

\section{Serum Levels of cTnIMean \pm SD and Prevalence of Cardiac Troponin-I}

The mean \pm SD of cardiac troponin-I in study participants was $17.896 \pm 9.796$. The overall prevalence of elevated cardiac troponin-I was $25.6 \%(21 / 82)$ among the study participants (Figure 1)

\section{Comparison of $\mathrm{cTnl}$ in Patients Taking Different Anti-Diabetic Drugs}

Patients who were taking metformin medication alone showed a significant mean decrease in serum cTn-I compared to the group on insulin alone (11.654 \pm 1.6795 versus $20.573 \pm 1.8402, \mathrm{P}<0.01)$. Similarly, patients who were taking metformin medication alone showed a significant mean decrease in serum cTn-I compared to patients who were on metformin and glibenclamide combination, (11.654 1.6795 versus $21.094 \pm 2.4062, \mathrm{P}<0.01$ vs). Patients receiving metformin alone also had numerically decreased mean of cardiac troponin-I compared to individuals on a combination of insulin and metformin $(11.654 \pm 1.6795$ versus $19.514 \pm 2.2249)$ but it was not

\section{Serum cTnl level}

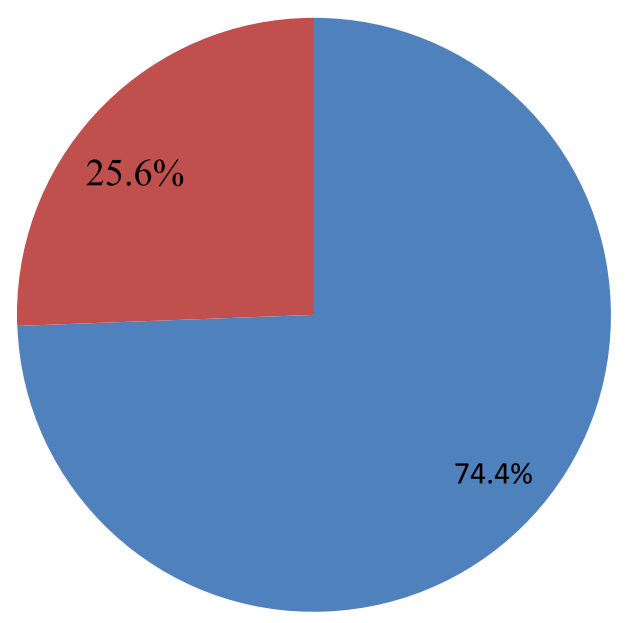

normal

Elevated

Figure I Prevalence of elevated cTnl among adult T2DM patients on chronic follow up at JMC, Jimma, 2020. statistically significant $(p>0.05)$. On the other hand, patients who were taking insulin and metformin combination showed a numerical decrease in cardiac troponin-I as compared to patients on insulin alone $(19.524 \pm 2.2249$ versus $20.573 \pm 1.8402$ ) as well as on metformin and glibenclamide combination (19.514 \pm 2.2249 versus $21.094 \pm 2.4062)$ but still there was no statistical significance (Figure 2 and Table 1).

After determining the presence of differences in mean value of serum cardiac troponin-I according to the type of anti-diabetic drugs, the Tukey HSD post hoc analysis was conducted and showed that there was statistically significant difference in mean serum cardiac troponin-I between patients who were taking insulin monotherapy and metformin monotherapy and, also between patients who were on metformin monotherapy versus metformin and glibenclamide at $\mathrm{P}<0.05$ (Table 1)

\section{Discussion}

Diabetic patients often exhibit silent cardiac dysfunction which is detectable only in the latter stage of the disease. Even $\approx 50 \%$ of individuals with well-controlled diabetes mellitus, asymptomatic and normotensive patients are considered to exhibit some degree of cardiac dysfunction. ${ }^{33}$ High sensitive cardiac markers such as cardiac troponin-I is currently considered as the gold standard diagnostic tool of myocardial injury. ${ }^{10}$

In this study, $25.6 \%(21 / 82)$ of the study participants showed elevated cardiac troponin-I. This was in line with a study conducted in the USA where cardiac troponin was elevated in $26 \%$ of type- 2 diabetic patients. ${ }^{20}$ But it was higher than the study in India that reported the prevalence of elevated cardiac troponin-I as $20.7 \%$ in acute stroke patients ${ }^{34}$ and the study conducted in the UK that reported the prevalence of elevated cardiac troponin-I (12.4\%) among patients who were attending emergency departments without acute coronary syndrome. ${ }^{35}$ On the other hand, the finding of this study was lower than the study in USA that reported $32.3 \%$ of elevated cardiac troponin-I in patients with hypertensive emergency. ${ }^{36}$ The discrepancy may be due to the difference in the study population, sample size and study design.

Multivariable logistic regression analysis revealed that type-2 diabetic patients on metformin were significantly associated with decreased cardiac troponin-I (AOR= 0.015, $\mathrm{P}<0.05)$ compared to patients who were taking metformin plus glibenclamide (Table 1). 


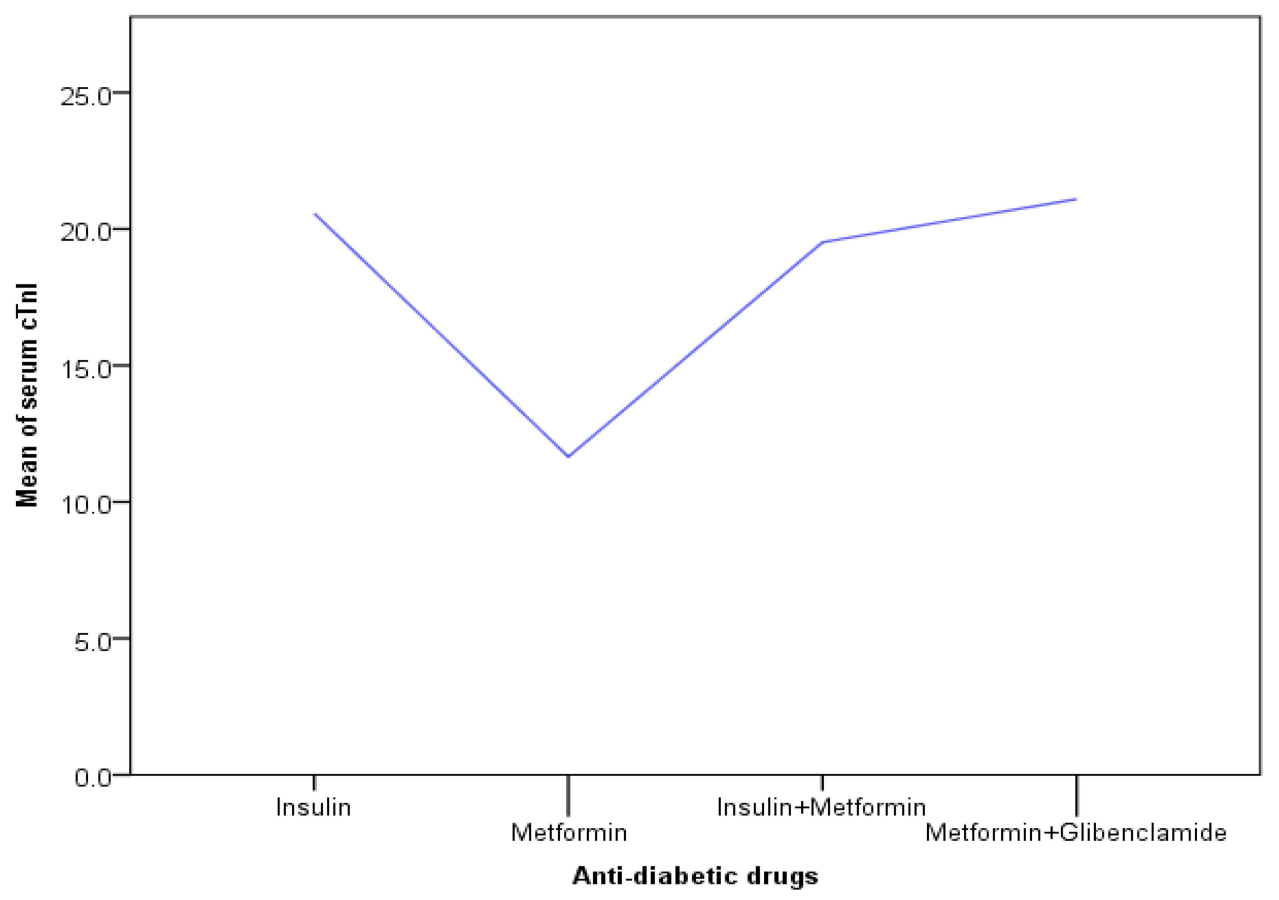

Figure 2 Mean of serum cTnl level among type-2 diabetic patients on different anti-diabetic drugs at JMC, Jimma, 2020.

The study conducted by Arif and Al-Ezzi on an animal model found a similar result; significant reduction of serum level of troponin-I in the metformin treated group. $^{32}$ Another study conducted in Argentina reported lower cardiac troponin in patients who were taking metformin as well as glibenclamide compared to control. This finding was also consistent with our study regarding metformin. ${ }^{21}$ Concerning glibenclamide, our study was inconsistent with this finding; the reason may be explained by the fact that our study was a comparison of metformin with glibenclamide containing treatment category, not with control a group. A similar result regarding the negative association of metformin with cardiac troponin was reported by Lexis et al. ${ }^{22}$ Our finding was also supported by the study that compared the effect of metformin versus sulfonylurea/insulin treatments on a 10-year follow up period which found a $33 \%$ reduction in myocardial infarction risk in contrary to $15 \%$ decrease by the latter. $^{24}$

Table I Tukey HSD Post Hoc Analysis for Pair-Wise Multiple Comparison of cTnl in Patients on Different Types of Anti-Diabetic Drugs at JMC, Jimma, 2020

\begin{tabular}{|c|c|c|c|c|}
\hline (I)Type of Drug & (J) Type of Drug & Mean Difference (I-J) & Std. Error & Sig. \\
\hline \multirow[t]{3}{*}{ Insulin } & Metformin & 8.9189 & 2.5703 & 0.005 \\
\hline & Insulin+Metformin & 1.0588 & 1.0588 & 3.0100 \\
\hline & Metformin+glibemclamide & -0.5214 & -0.5214 & $2.784 I$ \\
\hline \multirow[t]{3}{*}{ Metformin } & Insulin & -8.9189 & 2.5703 & 0.005 \\
\hline & Insulin+Metformin & -7.8601 & 3.0536 & 0.057 \\
\hline & Metformin+glibemclamide & -9.4403 & 2.8312 & 0.007 \\
\hline \multirow[t]{3}{*}{ Insulin+Metformin } & Insulin & -1.0588 & 3.0100 & 0.985 \\
\hline & Metformin & 7.8601 & 3.0536 & 0.057 \\
\hline & Metformin+glibemclamide & -1.5802 & 3.2356 & 0.961 \\
\hline \multirow[t]{3}{*}{ Metformin+glibemclamide } & Insulin & 0.5214 & $2.784 I$ & 0.998 \\
\hline & Metformin & 9.4403 & 2.8312 & 0.007 \\
\hline & Insulin+Metformin & 1.5802 & 3.2356 & 0.961 \\
\hline
\end{tabular}


A systematic and meta-analysis study also revealed that metformin was effective in reducing the incidence of cardiovascular events compared to sulfonylureas. ${ }^{25}$

Metformin may reduce cardiac troponin-I through several mechanisms, but the exact mechanism remains unclear. $^{26}$ Metformin phosphorylates AMP-activated kinase is related to many targets related to reduction the of I/R injury, and this activates Reperfusion Injury Salvage Kinase (RISK) path including PI3K and AKt pathways, increasing tumor suppressor gene $\mathrm{p} 53$, inactivation of mammalian target of rapamycin (mTOR) and activation of endothelial nitric oxide synthase (eNOS). ${ }^{27}$

The other mechanism of action of metformin may be through enhancing glucose utilization of the heart by increasing glucose transporters (GLUT-1 and GLUT-4). Moreover, metformin activates the adenosine receptor via increased intracellular formation of adenosine, possibly reducing MI size. ${ }^{28}$ On the other hand, metformin is associated with a decrease in Dipeptidyl peptidase-4 activity and an increase in circulating levels of glucagon-like peptide-1, which are associated with a reduction of $\mathrm{MI}$ size. $^{23,29}$ Overall, these metformin-induced changes in myocardial gene and energy program, are due to the fact that of activation of AMPK. ${ }^{30,31}$ In addition, metformin also has several beneficial effects such as antiinflammatory benefits, reduces oxidative stress, lowers endothelial dysfunction, and reduces hypertension. ${ }^{24}$

This finding did not agree with the study done by Basnet et al that reported no significant association between metformin treatment and MI. ${ }^{23}$ This disagreement may be due to the evaluation of troponin in the latter study group was carried out in a population which already developed myocardial infarction.

\section{Limitations of the Study}

Since the study was conducted exclusively in one hospital and the sample size was relatively small, our findings might not well represent all cases and be sufficient for generalization.

Besides, since the study was cross sectional, there might be the possibility of residual confounding variables as in observational studies, and because the exposure and outcome were assessed concomitantly and thereby it did not show temporal relation between cause and effect.

\section{Conclusion}

In the current study, about one fourth of type-2 diabetic patients on follow up had elevated cardiac troponin-I.
Metformin significantly reduced serum cTnI levels compared to insulin monotherapy and metformin + glibenclamide combination therapy.

\section{Copyright/Ethics}

We confirm that this study was conducted in accordance with the Declaration of Helsinki.

\section{Acknowledgments}

The abstract of this paper is available at the Jimma University website: https://repository.ju.edu.et/handle/ $123456789 / 5756$ as a thesis work supervised by the corresponding and other authors in the Jimma University. This does not mean that the paper is published.

\section{Disclosure}

The authors report no conflicts of interest in this work.

\section{References}

1. World Health Organization. Classification of Diabetes Mellitus. Geneva;2019:5-7

2. American Diabetes Association. Older adults: standards of medical care in diabetes-2019. Diabetes Care. 2019;42(Supplement1): S139-47. doi:10.2337/dc19-S012

3. Ozougwu JC, Obimba KC, Belonwu CD, Unakalamba CB. The pathogenesis and pathophysiology of type 1 and type 2 diabetes mellitus. J Physiol Pathophysiol. 2013;4(4):46-57. doi:10.5897/ JPAP2013.0001

4. Raffield LM, Hsu FC, Cox AJ, Carr JJ, Freedman BI, Bowden DW. Predictors of all-cause and cardiovascular disease mortality in type 2 diabetes: Diabetes Heart Study. Diabetol Metab Syndr. 2015;7(1):58. doi:10.1186/s13098-015-0055-y

5. Sundström J, Ingelsson E, Berglund L, et al. Cardiac troponin-I and risk of heart failure: a Community-Based Cohort Study. Eur Heart J. 2009;30(7):773-781. doi:10.1093/eurheartj/ehp047

6. Welsh P, Preiss D, Hayward C, et al. The biomarkers for acute myocardial infarction and heart failure. Biomed Res Int. 2020;17 (2020):2754-2764.

7. Roever L, Resende ES, Roerver-Borges AS. Hypertroponinemia, structural cardiac disease, and stroke mortality. Am Heart Assoc. 2017;48(5):1134-1136.

8. Korff S, Katus HA, Giannitsis E. Differential diagnosis of elevated troponins. Heart. 2006;92(7):987-993. doi:10.1136/hrt.2005.071282

9. Bodor GS. Biochemical markers of myocardial damage. Ejifcc. 2016;27(2):95.

10. Zhong R, Wang H, Tao Z, et al. Multi-center study of the 99th percentile value of high-sensitivity troponin $\mathrm{i}$ in a normal healthy chinese population. Clin Investig (Lond). 2017;7(2):49-57. doi:10.4172/Clinical-Investigation.1000111

11. Gottlieb A, Yanover C, Cahan A, Goldschmidt Y. Estimating the effects of second-line therapy for type 2 diabetes mellitus: Retrospective Cohort Study. BMJ Open Diabetes Res Care. 2017;1 (5):1.

12. Chaudhury A, Duvoor C, Reddy Dendi VS, et al. Clinical review of antidiabetic drugs: implications for type 2 diabetes mellitus management. Front Endocrinol (Lausanne). 2017;8(6). doi:10.3389/ fendo.2017.00006 
13. Ogurtsova K, daRocha Fernandes JD, Huang Y, et al. IDF diabetes atlas: global estimates for the prevalence of diabetes for 2015 and 2040. Diabetes Res Clin Pract. 2017;1(128):40-50. doi:10.1016/j. diabres.2017.03.024

14. Zimmermann M, Bunn C, Namadingo H, Gray CM, Lwanda J. Experiences of type 2 diabetes in sub-Saharan Africa: a scoping review. Glob Health Res Policy. 2018;3(1):25. doi:10.1186/s41256-018-0082-y

15. Zekewos A, Loha E, Egeno T, Wubshet K, Merga Z. Prevalence of diabetes mellitus and associated factors in Southern Ethiopia: a Community Based Study. Ethiop J Health Sci. 2018;28 (4):451-460. doi:10.4314/ejhs.v28i4.11

16. De Rosa S, Arcidiacono B, Chiefari E, Brunetti A, Indolfi C, Foti DP. Type 2 diabetes mellitus and cardiovascular disease: genetic and epigenetic links. Front Endocrinol (Lausanne). 2018;9(2). doi:10.3389/fendo.2018.00002

17. Martín-Timón I, Sevillano-Collantes C, Segura-Galindo A, Del Cañizo-Gómez FJ. Type 2 diabetes and cardiovascular disease: have all risk factors the same strength? World J Diabetes. 2014;5 (4):444-470. doi:10.4239/wjd.v5.i4.444

18. Marín-Peñalver JJ, Martín-Timón I, Sevillano-Collantes C, Del Cañizo-Gómez FJ. Update on the treatment of type 2 diabetes mellitus. World J Diabetes. 2016;7(17):354-395. doi:10.4239/wjd. v7.i17.354

19. Abdissa SG, Deressa W, Shah AJ. Incidence of heart failure among diabetic patients with ischemic heart disease: a Cohort Study. BMC Cardiovasc Disord. 2020;12(20):1-9.

20. Tang O, Matsushita K, Coresh J, et al. 258-OR: use of high sensitivity cardiac troponin $\mathrm{T}$ and $\mathrm{I}$ for risk classification in type 2 diabetes. Diabetes. 2019;68(supplement):1. doi:10.2337/db19-258-OR

21. Joison AN, Baiardi GC. Effect of pharmacological treatment on cardiac biomarkers in patients with acute coronary syndrome of non-ST segment elevation with type-2 diabetes. Int $J$ Med Health Sci. 2016;5(1):32-37.

22. Lexis CP, Wieringa WG, Hiemstra B, et al. Chronic metformin treatment is associated with reduced myocardial infarct size indiabetic patients with ST-segment elevation myocardial infarction. Cardiovasc Drugs Ther. 2014;28(2):163-171. doi:10.1007/s10557013-6504-7

23. Basnet S, Kozikowski A, Makaryus AN, et al. Metformin and myocardial injury in patients with diabetes and ST-segment elevation myocardial infarction: a propensity score matched analysis. $J \mathrm{Am}$ Heart Assoc. 2015;4(10):1-7.

24. Vesa CM, Popa L, Popa AR, et al. Current data regarding the relationship between type 2 diabetes mellitus and cardiovascular risk factors. Diagnostics. 2020;10(314). doi:10.3390/ diagnostics 10050314

25. Han Y, Xie H, Liu Y, Gao P, Yang X, Shen Z. Effect of metformin on all-cause and cardiovascular mortality in patients with coronary artery diseases: a systematic review and an updated meta-analysis Cardiovasc Diabetol. 2019;18(1):1-16. doi:10.1186/s12933-019. 0900-7
26. El Messaoudi S, Schreuder TH, Kengen RD, et al. Impact of metformin on endothelial ischemia-reperfusion injury in humans in vivo: a Prospective Randomized Open, Blinded-Endpoint Study. PLoS One. 2014;9(4):e96062. doi:10.1371/journal.pone.0096062

27. Maack C, Lehrke M, Backs J, et al. Heart failure and diabetes: metabolic alterations and therapeutic interventions: a state-of-the-art review from the translational research committee of the Heart Failure Association-European Society of Cardiology. Eur Heart J. 2018;39 (48):4243-4254. doi:10.1093/eurheartj/ehy596

28. Du J, Zhu M, Li H, Liang G, Li Y, Feng S. Metformin attenuates cardiac remodeling in mice through the Nrf2/Keap1 signaling pathway. Exp Ther Med. 2020;20(2):838-845. doi:10.3892/ etm.2020.8764

29. McCreight LJ, Bailey CJ, Pearson ER. Metformin and the gastrointestinal tract. Diabetologia. 2016;59(3):426-435. doi:10.1007/ s00125-015-3844-9

30. Wan X, Huo Y, Johns M, et al. 5'-AMP-activated protein kinaseactivating transcription factor 1 cascade modulates human monocytederived macrophages toatheroprotective functions in response to heme or metformin. Arterioscler Thromb Vasc Biol. 2013;33 (11):2470-2480. doi:10.1161/ATVBAHA.113.300986

31. Goldberg IJ, Eckel RH, McPherson R. Triglycerides and heart disease: still a hypothesis? Arterioscler Thromb Vasc Biol. 2011;31 (8):1716-1725. doi:10.1161/ATVBAHA.111.226100

32. Arif I, Al-Ezzi MI. The protective effect of metformin against doxorubicin induced cardiotoxicity in rabbits. Al-Mustansiriyah J Pharm Sci. 2019;19(1):54-56.

33. Marwick TH, Ritchie R, Shaw JE, Kaye D. Implications of underlying mechanisms for the recognition and management of diabetic cardiomyopathy. J Am Coll Cardiol. 2018;71(3):339-351. doi:10.1016/j.jacc.2017.11.019

34. Wrigley P, Khoury J, Eckerle B, et al. Prevalence of positive troponin and echocardiogram findings and association with mortality in acute ischemic stroke. Stroke. 2017;48(5):14561. doi:10.1161/ STROKEAHA.116.014561

35. Lee KK, Noaman A, Vaswani A, et al. Prevalence, determinants, and clinical associations of high-sensitivity cardiac troponin in patients attending emergency departments. Am J Med. 2018;132(1):110.e8110.e21. doi:10.1016/j.amjmed.2018.10.002

36. Afonso L, Bandaru H, Rathod A, et al. Prevalence, determinants, and clinical significance of cardiac troponin-i elevation in individuals admitted for a hypertensive emergency. J Clin Hypertens. 2011;13 (8):551-556. doi:10.1111/j.1751-7176.2011.00476.x

\section{Publish your work in this journal}

Research Reports in Clinical Cardiology is an international, peerreviewed, open access journal publishing original research, reports, editorials, reviews and commentaries on all areas of cardiology in the clinic and laboratory. The manuscript management system is completely online and includes a very quick and fair peer-review system. Visit http://www.dovepress.com/testimonials.php to read real quotes from published authors. 